\title{
Automatic Image Registration Technique of Remote Sensing Images
}

\author{
M. Wahed, Gh.S. El-tawel \\ Computer Science Department \\ Suez Canal University \\ Ismailia, Egypt
}

\author{
A. Gad El-karim \\ Mathematics Department \\ Suez Canal University \\ Alarish, Egypt
}

\begin{abstract}
Image registration is a crucial step in most image processing tasks for which the final result is achieved from a combination of various resources. Automatic registration of remote-sensing images is a difficult task as it must deal with the intensity changes and variation of scale, rotation and illumination of the images. This paper proposes image registration technique of multi-view, multi- temporal and multispectral remote sensing images. Firstly, a preprocessing step is performed by applying median filtering to enhance the images. Secondly, the Steerable Pyramid Transform is adopted to produce multi-resolution levels of reference and sensed images; then, the Scale Invariant Feature Transform (SIFT) is utilized for extracting feature points that can deal with the large variations of scale, rotation and illumination between images .Thirdly, matching the features points by using the Euclidian distance ratio; then removing the false matching pairs using the RANdom SAmple Consensus (RANSAC) algorithm. Finally, the mapping function is obtained by the affine transformation. Quantitative comparisons of our technique with the related techniques show a significant improvement in the presence of large scale, rotation changes, and the intensity changes. The effectiveness of the proposed technique is demonstrated by the experimental results.
\end{abstract}

Keywords - Image registration; Steerable Pyramid Transform; SIFT; RANSAC

\section{INTRODUCTION}

Image registration is a fundamental task in image processing used to match two or more images which are taken at different time, from different sensors or different viewpoints [1]. The present image registration methods can be generally divided into two broad categories: area-based and feature-based methods [2]. Area-based methods deal with the images without detecting salient features, and adopt optimization algorithms. These methods are substantial to the intensity distribution. The feature-based methods do not directly work with image intensity values, but, instead, use salient features extracted from two images, which has been shown to be more suitable for such situations that intensity changes and complicated geometric deformations are encountered. Therefore, these feature-based methods have been widely used in remote-sensing image registration. Feature-based image registration consists of five steps: preprocessing, feature selection, feature correspondence, transformation and resampling. Among them, feature selection, correspondence and transformation require numerous manipulation techniques, where in the most difficult one is the feature correspondence. If some correspondences are incorrect, they will produce an incorrect transformation function, which could yield totally wrong results, so a highly robust matching algorithm is needed. The process of image registration intersects with the following research areas: computer vision, pattern recognition, and remotely sensed data processing. In general, its applications can be divided into multi-view analysis, multi-temporal analysis and multimodal analysis according to the manner of the image acquisition.

In literature, there are several image registration techniques have been proposed, Xiangzeng and al. [3] Proposed multi-scale image registration technique based on steerable pyramid transform and Scale Invariant Feature Transform (SIFT) of remote sensing image. Nagham and al. [4] presented wavelet-based image registration technique that combined Scale Invariant Feature Transform (SIFT) with Mutual-Information (MI). Haidawati and al. [5] developed image registration approach based on a combination of Scale Invariant Feature Transform (SIFT), Belief Propagation (BP) for matching features and Random Sampling Consensus (RANSAC) adopted to filter out the mismatched points. Sang [6] introduced automatic coarse-to-fine image registration algorithm for satellite images, based on Haar Wavelet Transform (HWT) and the Speeded Up Robust Features (SURF) algorithm in the coarse registration, the normalized cross-correlation and RANdom SAmple Consensus (RANSAC) algorithm to achieve the fine registration. Mahmudul and al. [7] proposed a method to improve SIFTbased matching for multispectral image registration. Fatiha and al. [8] presented an efficient image registration algorithm that used the genetic algorithms and the cross-correlation similarity measure for matching within a multi-resolution framework based on the Non-Subsampled Contourlet Transform (NSCT).Yi and al. [9] presented an enhanced SIFT method for multi-spectral remote sensing image registration. Le and al. [10] developed a fully automatic and fast non-rigid image registration technique that coarsely aligned the input image to the reference image by automatically detecting their matching points by using the scale invariant feature transform (SIFT) method and an affine transformation model. Gang and Yun [11] introduced image registration technique, which is based on wavelet-based feature extraction technique, a normalized cross-correlation matching and relaxation-based image matching techniques. Leila and al. [12] presented efficient image registration algorithm of multi-temporal images with similar spectral responses based on modulus 
maxima of wavelet transform for point features extraction and a correlation based matching measure used in the matching process. Shirin and Kasaei [13] developed image registration method based on Contourlet Transform for extracting edge features from panchromatic satellite images and matching features by normalized cross-correlation.

In this paper we present automatic image registration technique of remote sensing image based on the Steerable Pyramid Transform and SIFT descriptors. This paper is organized as follows. Section 2 presents the proposed image registration technique. Experimental results and conclusions are given in Sections 3 and 4, respectively.

\section{Proposed Image Registration TechniQue}

In this section, we describe the proposed image registration technique which consists of six steps: preprocessing, decomposition by steerable pyramid transform, extract feature points using the Scale Invariant Feature Transform (SIFT), Find all matching pairs between two images, remove false matching pairs, perform affine transformation and resampling to perform image registration. The work flow of the proposed technique is shown in Fig.1.

\section{A. Preprocessing}

Given two input images (the reference image and the sensed image), applying median filtering to the reference image and the sensed image in order to enhance the two input images.

\section{B. Steerable Pyramid Transform}

The steerable pyramid transform is a linear multi-scale, multi-orientation image decomposition that provides a useful front-end for image processing and computer vision applications [14]. It has been developed in order to overcome the limitations of orthogonal separable wavelet decompositions that were popular for image processing. The "steerable filter" refers to a class of filters, in which a filter of arbitrary orientation can be synthesized as a linear combination of a set of "basis filters". For any function $f(x, y), f^{\theta}(x, y)$ is $f(x, y)$ rotated through an angle $\theta$ about the origin. We call $f(x, y)$ is steerable if it satisfies the following equation:

$f^{\theta}(x, y)=\sum_{j=1}^{M} k_{j}(\theta) f^{\theta j}(x, y)$.

Where $k_{j}(\theta)$ are the interpolation functions $j=(1, \ldots, M)$ .The basic functions of the steerable pyramid are directional derivative operators that come in different sizes and orientations, and the number of orientations may be adjusted by changing the derivative order. The structure of the steerable pyramid in the frequency domain is shown in Fig.2.The image is initially divided into high and low-pass sub-bands using filters $H_{0}(\omega)$ and $L_{0}(\omega)$. The low-pass branch is then further divided into oriented band-pass portions using filters $\left\{B_{0}(\omega), \ldots, B_{k}(\omega)\right\}$ which ensure that the representation is rotation invariant and lower-pass portion using filter

$L_{1}(\omega)$.this lower-pass sub-band is sub-sampled by a factor of 2 in the $X$ and $Y$ directions. In order to ensure translation- invariance, the outputs of the high-pass filter and of the bandpass filters are not sub-sampled. In addition, that portion of the signal, which is iteratively decomposed by the band-pass and the low-pass filters, does not contain the larger high frequency components and has been preprocessed by a low-pass filter, thus removing most aliased component, thus, to eliminate aliasing terms, $L_{1}(\omega)$ is constrained as:

$$
L_{1}(\omega)=0 \text { For }|\omega|>\frac{\pi}{2}
$$

The recursive construction of a pyramid is achieved by inserting a copy of the shaded portion of the System diagram in Fig.2 at the location of the solid circle. The steerable pyramid performs a polar-separable decomposition in the frequency domain, thus allowing independent representation of scale and orientation. In order to cascade the system recursively, there should be

$$
\left|L_{1}(\omega / 2)\right|^{2}=\left|L_{1}(\omega / 2)\right|^{2}\left[\left|L_{1}(\omega)\right|^{2}+\sum_{k=0}^{n}\left|B_{k}(\omega)\right|^{2}\right]
$$

In the proposed technique we apply the steerable pyramid transform to the two input images to level three with one orientation band-pass component. Fig.3 shows three level of steerable pyramid decomposition with one orientation bandpass filter for the reference image and the sensed image, respectively.

\section{SIFT Feature Point Extraction Algorithm}

SIFT algorithm was proposed in [15] as a method to extract and describe feature points, which is robust to scale, rotation and change in illumination. There are four steps to implement the SIFT algorithm:

1) Scale-space Extrema Detection: The first stage searches over scale space using a Difference of Gaussian (DoG) function to identify potential interest points that are invariant to scale and orientation. The scale space of an image is defined as a function $L(x, y, \sigma)$, which is produced from the convolution of a variable-scale Gaussian $G(x, y, \sigma)$ with an input image $I(x, y)$ :

$L(x, y, \sigma)=G(x, y, \sigma) * I(x, y)$,

$G(x, y, \sigma)=\frac{1}{2 \pi \sigma^{2}} e^{-\left(x^{2}+y^{2}\right) / 2 \sigma^{2}}$

To efficiently detect stable key-point locations in scale space using scale-space extrema in the difference-of-Gaussian function convolved with the image, $D(x, y, \sigma)$ which can be computed from the difference of two nearby scales separated by a constant multiplicative factor $k$ :

$$
\begin{aligned}
D(x, y, \sigma)= & (G(x, y, k \sigma)-G(x, y, \sigma)) * I(x, y) \\
& =L(x, y, k \sigma)-L(x, y, \sigma) .
\end{aligned}
$$

2) Feature Point Localization: The location and the scale of each candidate point are determined and the feature points are selected based on measures of stability this information allows points to be rejected that have low contrast (and are therefore sensitive to noise) or are poorly localized along an edge. 


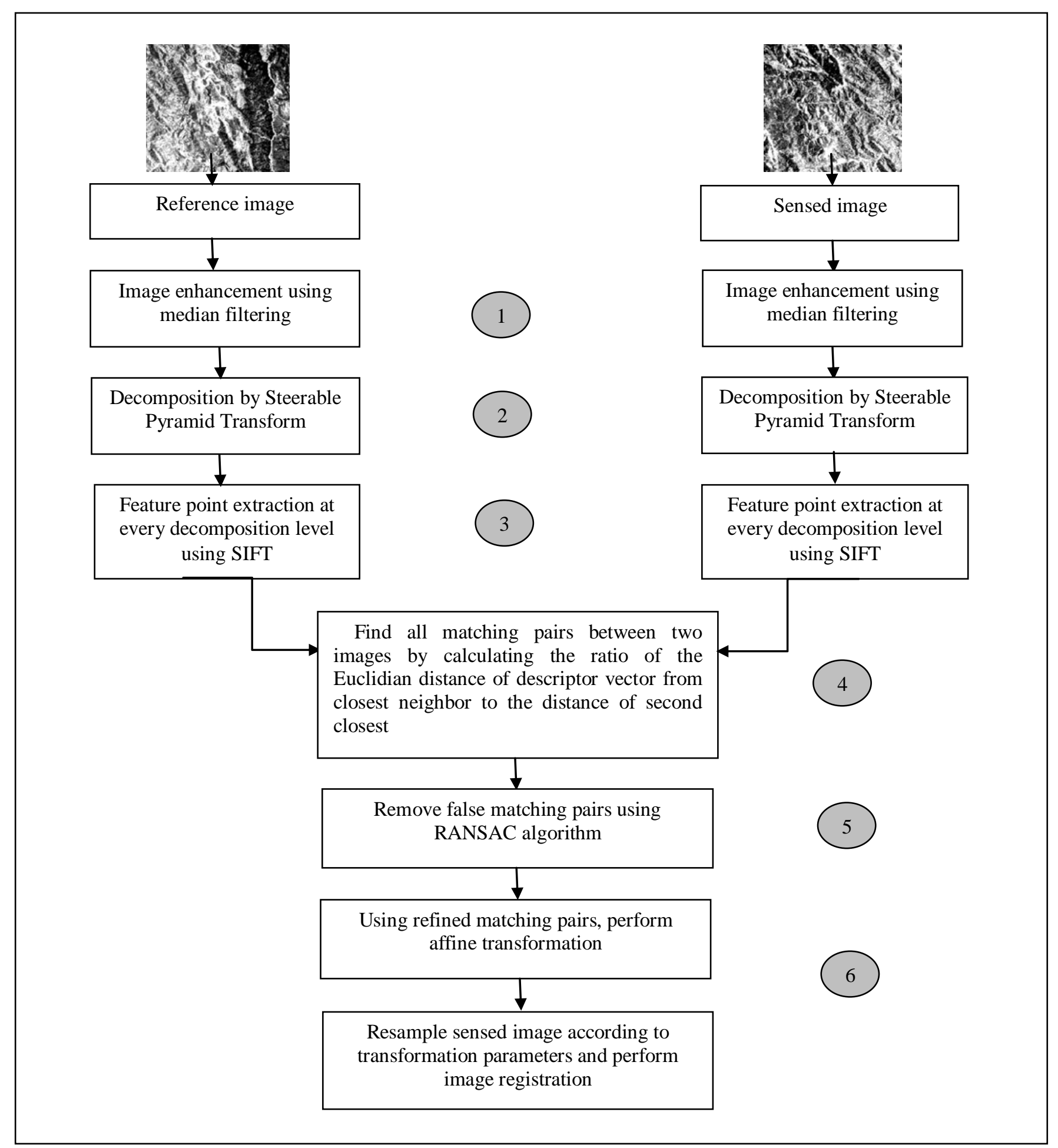

Fig. 1. Work Flow of the Proposed Technique 


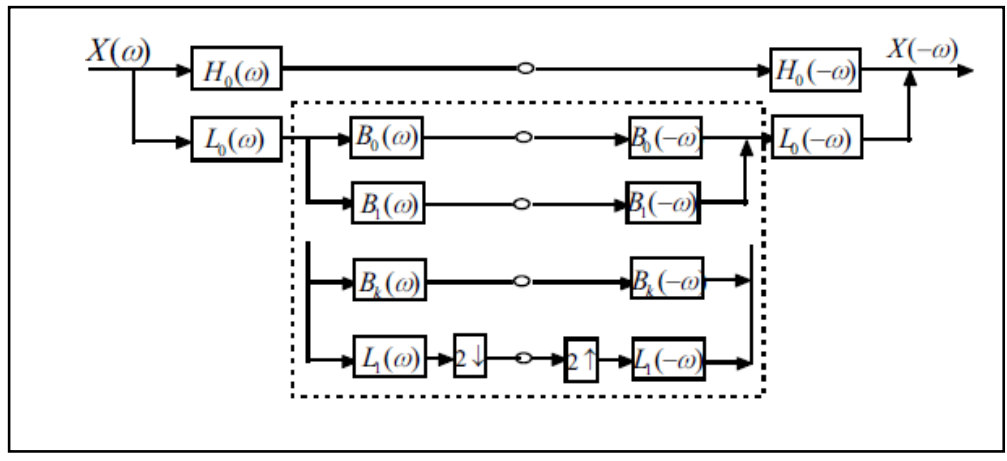

Fig. 2. System diagram for first derivative Steerable Pyramid

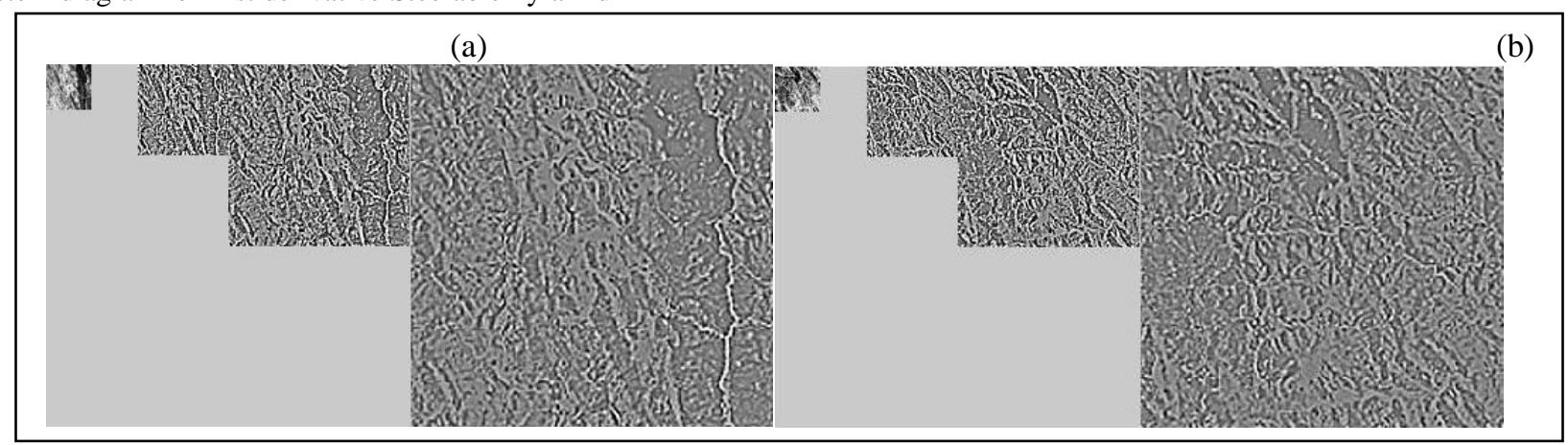

Fig. 3. Steerable Pyramid decomposition (l=3) for (a) reference image and (b) sensed image

3) Orientation Assignment: One or more orientations are assigned to each feature point location based on local image gradient directions. For each image sample at this scale $L(x, y)$, the gradient magnitude $m(x, y)$, and orientation $\theta(x, y)$ are precomputed using pixel differences:

$$
\begin{gathered}
m(x, y)=\sqrt{(L(x+1, y)-L(x-1, y))^{2}+(L(x, y+1)-L(x, y-1))^{2}}(7) \\
\theta(x, y)=\tan ^{-1}((L(x, y+1)-L(x, y-1)) /(L(x+1, y) \\
-L(x-1, y)))
\end{gathered}
$$

4) Feature Point Descriptor: A feature descriptor is created by first computing the gradient magnitude and orientation at each image sample point in a region around the feature point location, as shown on the left of Fig.4.These are weighted by a Gaussian window, indicated by the overlaid circle. These samples are then accumulated into orientation histograms summarizing the contents over $4 \times 4$ sub-regions, with 8 orientation bins. So each feature point has a 128element feature as shown on the right, with the length of each arrow corresponding to the sum of the gradient magnitudes near that direction within the region. The SIFT feature point extraction for the reference image and the sensed image is shown in Fig.5 and Fig.6.

\section{The proposed Feature Points Matching Using Structural Information}

SIFT algorithm at first detects feature points in scalespace, feature points with low contrast and located at edges are discarded. Then a 128-element feature descriptor is generated for each feature point using statistics of the gradient directions which are scale and rotation invariant. These descriptors are used to find the corresponding feature points by calculating the ratio of the Euclidian distance of descriptor vector from closest neighbor to the distance of second closest. To illustrate the issue, we show an example in Fig.7. SIFT matching is applied to images $A$ and $B$. The bold line shows a pair $(a, c)$ of matched featured points in the two images. The dotted line shows the best match $e$ of another feature point $b$ in image $A$, while the correct match should be point $d$. In the proposed technique $e$ is not selected as a matched feature point for $b$ because the spatial distance between points $c$ and $e$ is too large. Feature points $a$ and $c$ are matched while the counterpart for neighboring feature point $b$ cannot be decided because the SIFT descriptors for points $d$ and $e$ are almost equally different from the SIFT descriptor of point $b$. This problem is made worse by the fact that a lot of similar descriptors can be found in typical remote sensing images. The idea to solve this problem is as follows. Still considering the example in Fig.7, assume points $a$ and $c$ are already matched with high confidence that the match is correct. We can predict that the feature points around $a$ (shown in the circular window) can be found around $c$. So, for point $b$ we only search the neighborhood of point $c$ for a matching descriptor, which results in a correct match at point $d$. For better matching accuracy we suggest modifications to SIFT matching by imposing a threshold on the Euclidian distance ratio as follows: A descriptor $D 1(i, j)$ of feature point $a$ in image $A$ (reference image) is matched to a descriptor $D 2(i, j)$ of feature point $c$ in image $B$ (sensed image) only if the Euclidian distance $d(D 1, D 2)$ multiplied by threshold (set 
to 1.5) is not greater than the distance of $D 1$ to all other descriptors. SIFT matching result between the feature points of the reference image and the sensed image is shown in Fig.8.

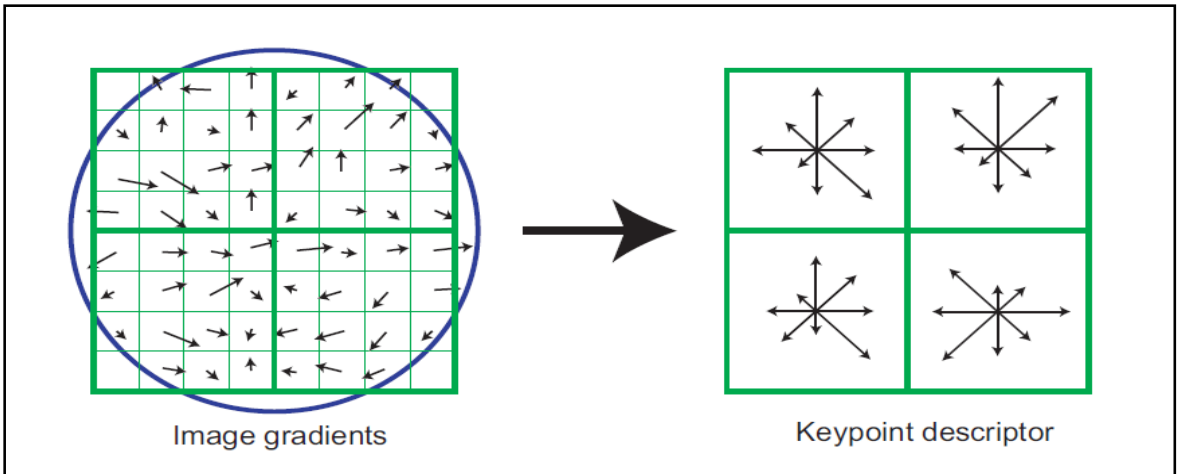

Fig. 4. Feature descriptor creation

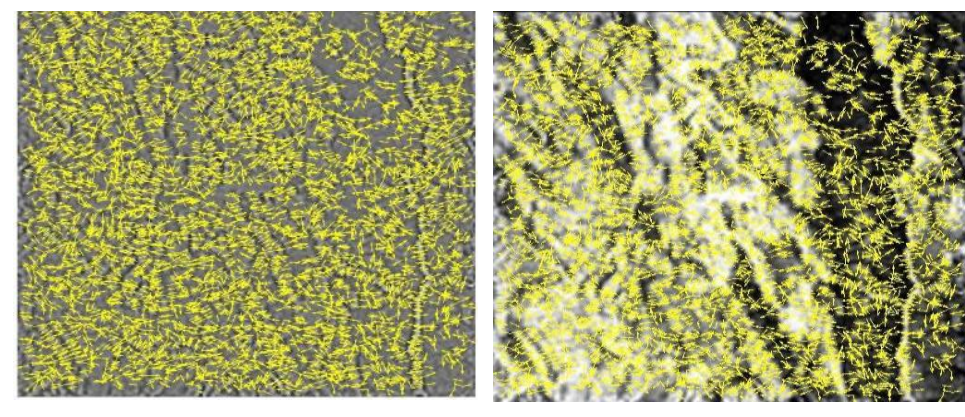

Fig. 5. Feature points returned by SIFT for reference image

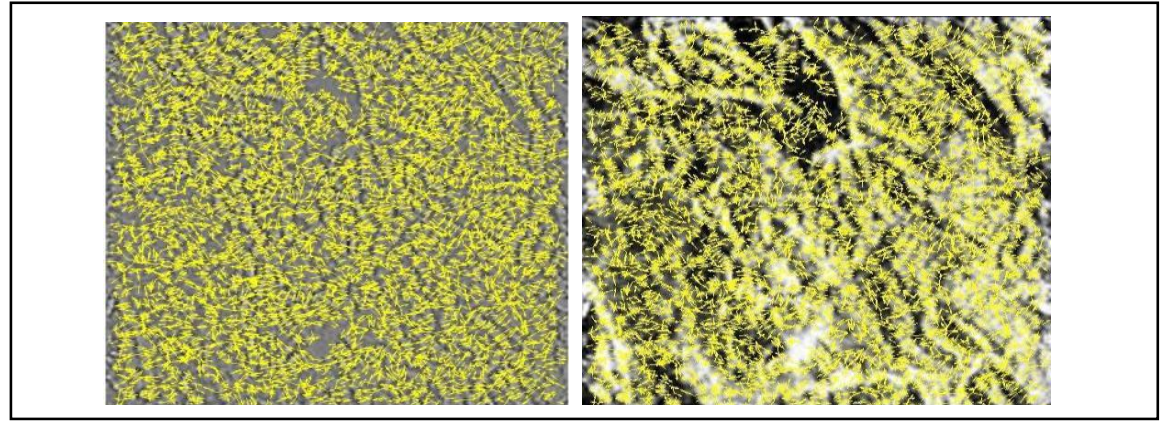

Fig. 6. Feature points returned by SIFT for sensed image

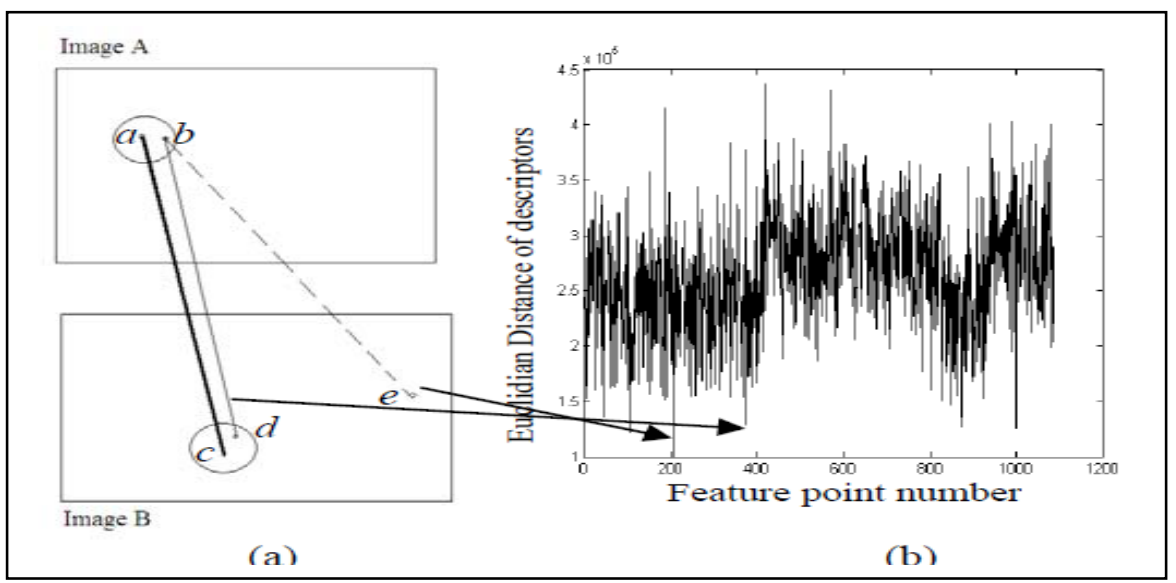

Fig. 7. a) Feature point matching results $a$ to $c$ points as correct match but no match found for b. (b) Euclidian distance of the descriptors of all the feature point on image $B$ for the feature point $b$ on image $A$. 


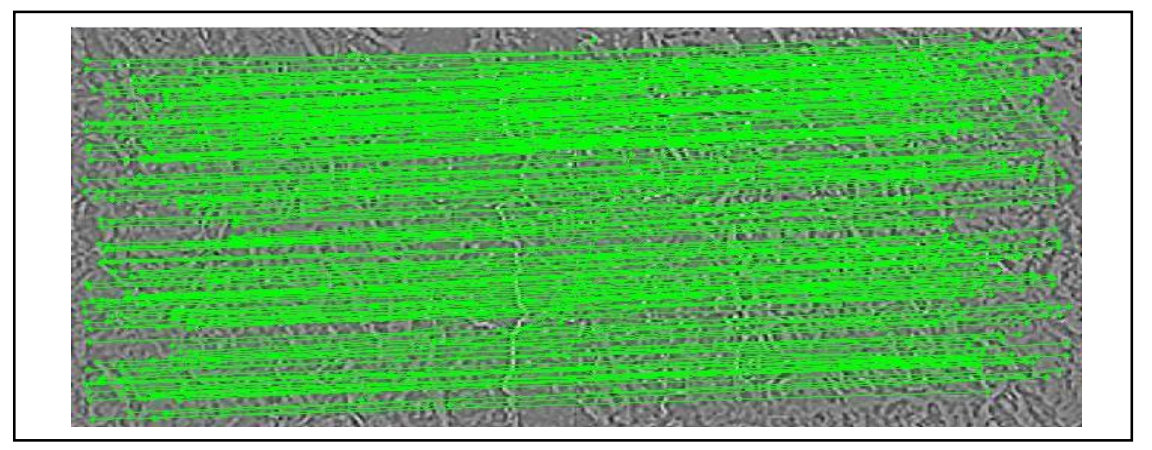

Fig. 8. Matching result between the reference image and sensed image in steerable domain.

\section{E. RANSAC (Random Sample Consensus) Algorithm}

In general there are all kinds of photometric and geometric transformations that can occur between two views of a scene. This means normalized cross-correlation will sometimes generate spurious correspondences. To robustly fit a model to the correspondences, we need to overcome the effect of these outliers. The RANdom SAmple Consensus (RANSAC) algorithm proposed by Martin and Robert [16] is a general parameter estimation approach designed to cope with a large proportion of outliers in the input data. There are two types of samples: contaminated, those that contain at least one outlier, and uncontaminated (all-inlier or outlier-free) samples. Only the latter ones are of interest, as the model parameters computed from data points including outliers are arbitrary. The number of iterations $N$ is chosen high enough to ensure that the probability $p$ (usually set to 0.99 ) that at least one of the sets of random samples does not include an outlier. Let $u$ represent the probability that any selected data point is an inlier and $v=1-u$ the probability of observing an outlier, $N$ iterations of the minimum number of points denoted $m$ are required, where

$$
\begin{aligned}
& 1-p=\left(1-u^{m}\right)^{N} \\
& \text {, and thus with some manipulation, } \\
& N=\frac{\log (1-p)}{\log \left(1-(1-v)^{m}\right)}
\end{aligned}
$$

A RANSAC algorithm provides a general technique for model fitting in the presence of outliers and consists of the following steps:

1) Choose a model.

2) Determine the minimal number of points needed to specify the model.

3) Define a threshold on the inlier count.

4) Fit the model to a randomly selected minimal subset
5) Apply the transformation to the complete set of points and count inliers.

6) If the number of inliers exceeds the threshold, flag the fit as good and stop.

7) Otherwise repeat steps 4 to 6 .

In our technique we apply RANSAC algorithm to the putative correspondences to remove false matching point pairs, which are consistent with this estimate because many of the putative correspondences obtained in the previous step are incorrect.

\section{F. Perform Affine Transformation and Resampling}

Given the refined matching point pairs, build the mapping function and get the affine transformation parameters to resample the sensed image and perform image registration.

\section{EXPERMENTAL RESULTS AND EVALUATION}

\section{A. Data Sets}

The proposed technique is tested for ten different sets of remote sensing images. We present three different sets of images. The first set of images is Landsat TM images from different bands (12-band 0 and 8) (Fig. 9(a) and (b)) with large rotation variation, which are used to show the implementation and accuracy of our algorithm. Images of the second set are Agricultural images from Landsat TM (band 5) of Amazon region acquired at different times (Fig.10 (a) and Fig. 10(b)) .The third data set are QuickBird panchromatic and nearinfrared band images (Fig.11 (a) and (b)) with large scale difference derived from Digital Globe, Inc; acquired on Jul. 4, 2005 over Boulder, USA . The near-infrared band has been shown to be effective for estimating moisture content and plant biomass in the 760-900 $\mathrm{nm}$ wavelength range. In addition, the panchromatic and multispectral images have 0.6 $\mathrm{m}$ and $2.4 \mathrm{~m}$ spatial resolution at nadir, respectively. Here, the near infrared band image of low spatial resolution is registered to the high resolution panchromatic image. The feature points information of the three data sets are shown in Table 1. 


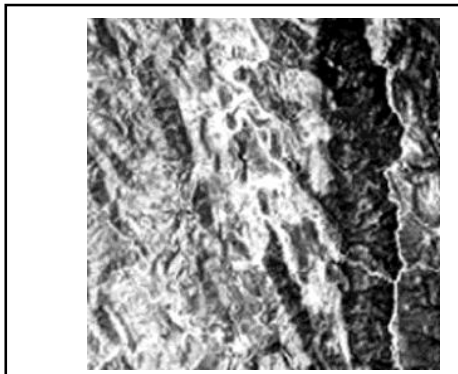

(a)

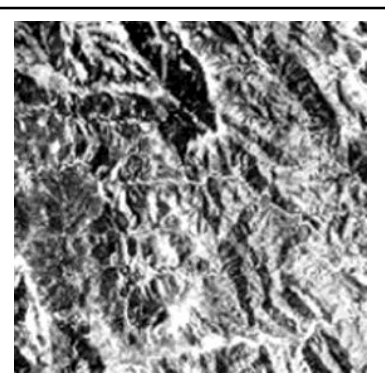

(b)

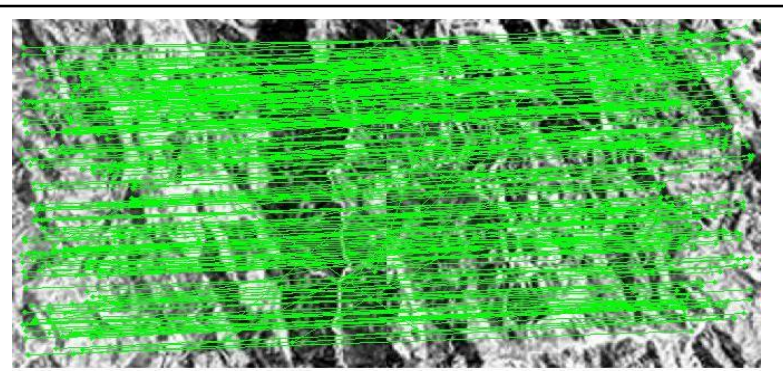

(c)

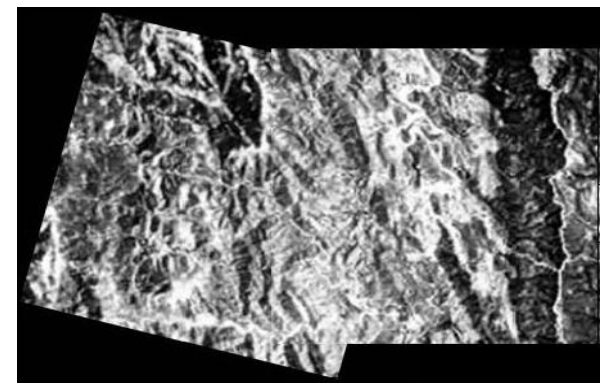

(d)

Fig. 9. Reference image, sensed image, matching results, and registration results (Landsat TM images). (a) The reference image (Landsat TM 12-Band 0, 512 by 512); (b) the sensed image (Landsat TM 12 -Band 8, 512 by 512); (c) the matching results of (a) and (b); (d) the registration results of (a) and (b).

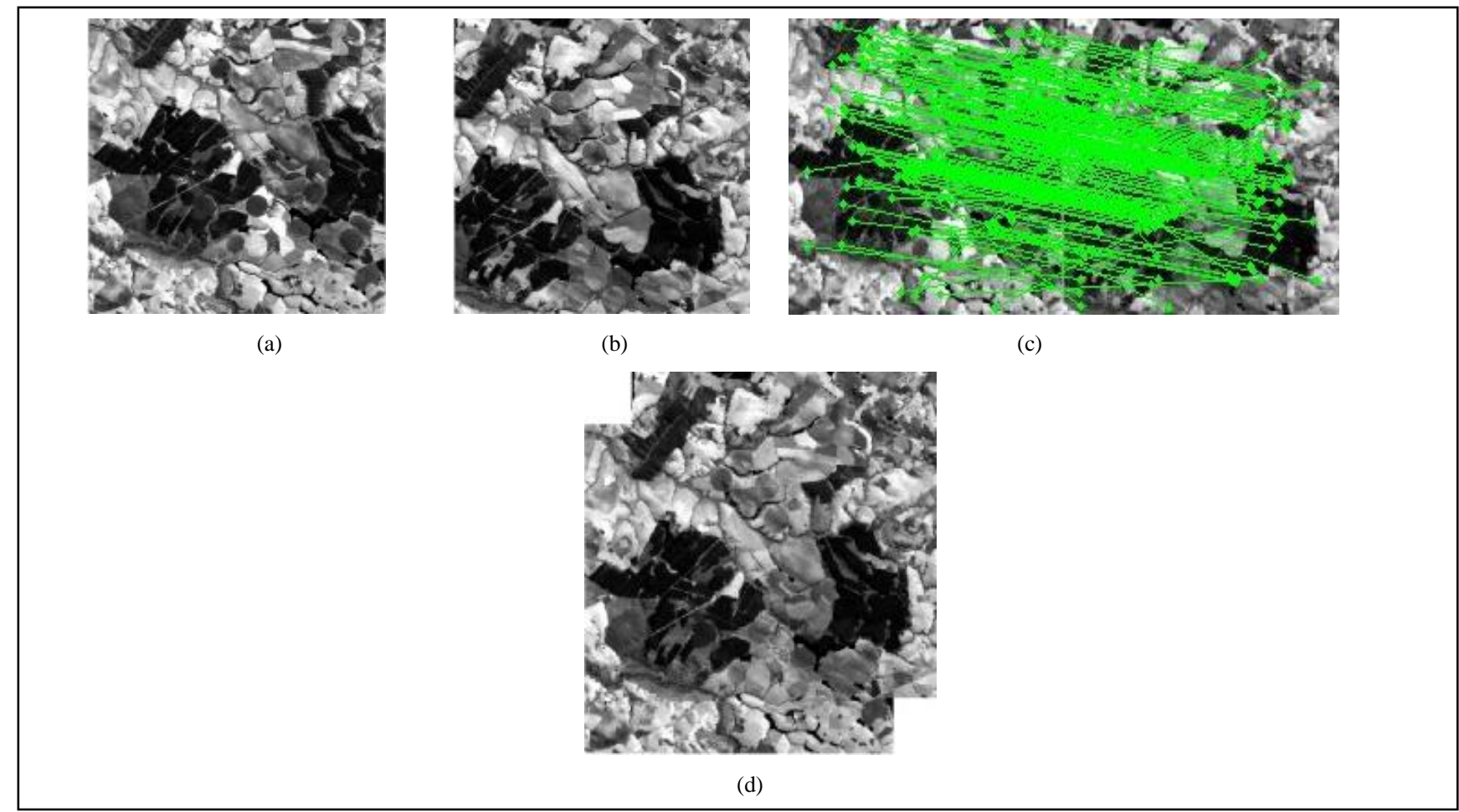

Fig. 10. Agricultural images from Landsat TM (band 5). (a) The reference image (Landsat TM 400 by 400 acquired on September 9, 1990); (b) the sensed image (Landsat TM 400 by 400 acquired on July 18, 1994.); (c) the matching results of (a) and (b); (d) the registration results of (a) and (b). 


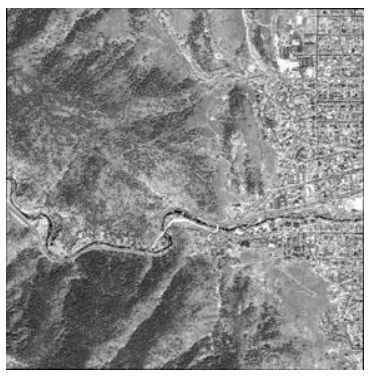

(a)

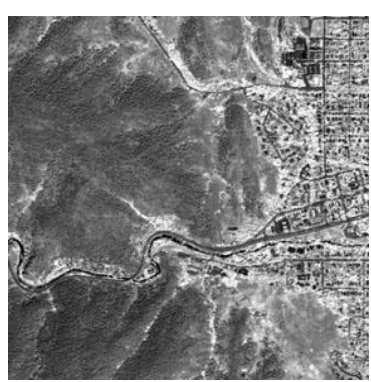

(b)

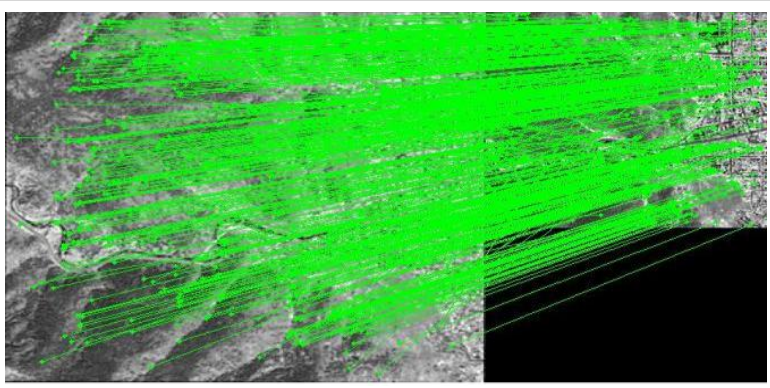

(c)

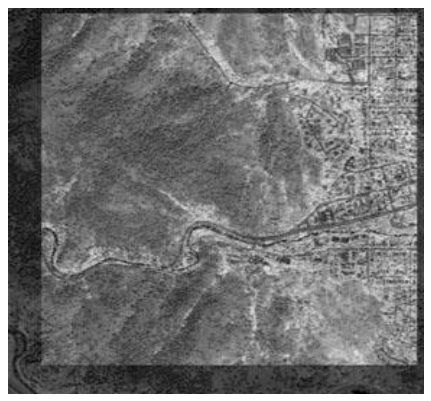

(d)

Fig. 11. Reference image, sensed image, matching results, and registration results of QuickBird panchromatic and near-infrared images (Image courtesy of Digital Globe) (a) The reference image (QuickBird panchromatic, 2048 by 2048); (b) the sensed image (QuickBird near-infrared, 937 by 915); (c) the matching results of (a) and (b); (d) the registration results of (a) and (b).

TABLE I. FEATURE POINTS INFORMATION (FIG.9-11)

\begin{tabular}{|c|c|c|c|c|c|c|}
\hline \multirow[b]{3}{*}{ Data sets } & \multicolumn{6}{|c|}{ Feature points information } \\
\hline & \multirow[t]{2}{*}{ Level no. } & \multirow[t]{2}{*}{ Image size(pixels) } & \multicolumn{2}{|c|}{ Number of feature points } & \multirow{2}{*}{$\begin{array}{l}\text { Number of initial } \\
\text { matched pairs }\end{array}$} & \multirow{2}{*}{$\begin{array}{l}\text { Number of refined } \\
\text { matched pairs using } \\
\text { RANSAC }\end{array}$} \\
\hline & & & Reference image & Sensed image & & \\
\hline \multirow[b]{2}{*}{ Fig .9 } & 1 & $512 \times 512$ & 6571 & 7502 & 244 & 161 \\
\hline & 2 & $256 \times 256$ & 3285 & 3498 & 420 & 270 \\
\hline \multirow[b]{2}{*}{ Fig .10 } & 1 & $400 \times 400$ & 4313 & 4192 & 174 & 108 \\
\hline & 2 & $200 \times 200$ & 1581 & 1519 & 169 & 102 \\
\hline \multirow{4}{*}{ Fig .11 } & 1 & $2048 \times 2048$ & 19091 & 13261 & 578 & 505 \\
\hline & & $937 \times 915$ & & & & \\
\hline & 2 & $1024 \times 1024$ & 16538 & 10137 & 1630 & 1553 \\
\hline & & $465 \times 457$ & & & & \\
\hline
\end{tabular}

\section{B. Evaluation}

In order to evaluate the proposed image registration technique; First we apply the proposed technique to the three sets of images. Second, we compare our technique with other two related techniques on the accuracy of matching and registration. To evaluate the matching result between the two input images, Assume that the transformation between the point $\left(x_{i}, y_{i}\right)$ in the sensed image and its corresponding point $\left(X_{i}, Y_{i}\right)$ in the reference image is affine transformation, we can use the root mean square error (RMSE) :
$R M S E=\left(\frac{\sum_{i=1}^{m}\left[\left(a x_{i}+b y_{i}+c-X_{i}\right)^{2}+\left(d x_{i}+e y_{i}+f-Y_{i}\right)^{2}\right]}{m}\right)^{1 / 2}$

Where $m$ means the total number of matching points; $(a, b, c, d, e, f)$ are affine transformation parameters. The transformation parameters between the two input images of the three data sets and their root mean square error (RMSE) are shown in Table 2.

The test three sets of images include large rotation, translation, scale, and intensity changes. We use Fig.9 (a) as the reference image and Fig.9 (b) as the sensed image. Fig.9 
(c) and (d) shows the matching results and the registration results, respectively. In the second data set we use two Landsat TM (band5) images with translation differences; Fig.10 (a) and Fig.10 (b), are used as the reference image and the sensed image, respectively. Fig.10 (c) shows the matching results and Fig. 10 (d) shows the registration results .Fig.11 (a) and (b) are QuickBird panchromatic and near-infrared band images with large scale variations and intensity changes, which is used as the reference image and the sensed image, respectively. The matching results and registration results are shown in Fig.11 (c) and (d), respectively. To compare the registration accuracy, we consider the root mean square error of intensity ( $R M S E 1$ ), the correlation (corr) between the overlapping areas of registered image pairs and Peak Signal to Noise Ratio $(P S N R)$, which are defined as follows:

$$
\begin{aligned}
R M S E 1 & =\left(\frac{\sum_{m, n \in R}\left(I_{m n}-J_{m n}\right)^{2}}{N}\right)^{\frac{1}{2}} \\
\operatorname{corr} & =\frac{\sum_{m, n \in R}\left(I_{m n}-\bar{I}_{R}\right)\left(J_{m n}-\bar{J}_{R}\right)}{\sqrt{\left(\sum_{m, n \in R}\left(I_{m n}-\bar{I}_{R}\right)^{2}\right)\left(\sum_{m, n \in R}\left(J_{m n}-\bar{J}_{R}\right)^{2}\right)}}
\end{aligned}
$$

TABLE II.

THE TRANSFORMATION PARAMETERS AND THEIR RMSE (FIG.9-11)

\begin{tabular}{|l|c|c|c|c|c|c|c|}
\hline \multirow{2}{*}{ Data sets } & \multicolumn{7}{|c|}{ Transformation Parameters and RMSE } \\
\cline { 2 - 8 } & $\boldsymbol{a}$ & $\boldsymbol{b}$ & $\boldsymbol{c}$ & $\boldsymbol{d}$ & $\boldsymbol{e}$ & $\boldsymbol{f}$ & RMSE \\
\hline Fig .9 & 0.9626 & -0.2551 & -10.7779 & 0.2650 & 0.9757 & 264.9061 & 0.11 \\
& & & & & & & \\
\hline Fig .10 & 0.9872 & -0.0006 & 69.6568 & 0.0005 & 1.0077 & -60.8548 & 0.33 \\
\hline Fig .11 & 0.9914 & -0.0001 & -17.7040 & 0.0002 & 1.0276 & -93.5854 & 0.17 \\
\hline
\end{tabular}

TABLE III. The ACCURACY OF MATCHING RESUltS AND REgistration RESUlts (FIG.9-11)

\begin{tabular}{|c|c|c|c|c|}
\hline \multirow{2}{*}{ Data sets } & \multicolumn{4}{|c|}{$\begin{array}{c}\text { Matching error, Registration error, } \\
\text { Correlation and Peak Signal to Noise Ratio }\end{array}$} \\
\cline { 2 - 5 } & $\boldsymbol{R M S E}$ & $\boldsymbol{R M S E 1}$ & corr & PSNR \\
\hline Fig.9 & 0.11 & 11.12 & 0.99 & 32.47 \\
\hline Fig.10 & 0.33 & 4.08 & 0.87 & 35.91 \\
\hline Fig.11 & 0.17 & 2.71 & 0.89 & 39.47 \\
\hline
\end{tabular}

$$
P S N R=20 \log \frac{255}{R M S E 1}
$$

Where $I$ is the reference image, $J$ is the registered image, $R$ is the overlapping area between $I$ and $J$, and $N$ is the number of pixels in $R$. The accuracy of matching results and registration results for Fig.9, Fig.10, and Fig.11 are shown in Table3. Fig.12 shows the registration accuracy in four resolution levels for Fig.9.

Experiment have been carried out on the first set of images (Landsat TM images) in order to compare the registration accuracy of the proposed technique against other image registration techniques (Xiangzeng and al. [3]) and (Le and al. [10]); we consider RMSE, RMSE1, corr . RMSE1 is smaller and the corr is the larger which shows higher accuracy of registration. The comparisons of accuracy of matching results and registration results for Fig.9 are shown in Fig.13. From Fig.13, we can see both that RMSE and RMSE1 of the proposed technique are the smallest and corr of the proposed technique is the largest. From the above experimental results, we can see that the proposed technique performs better than the other two techniques when large scale variations, rotation, translation, and intensity changes exist between the two input image 


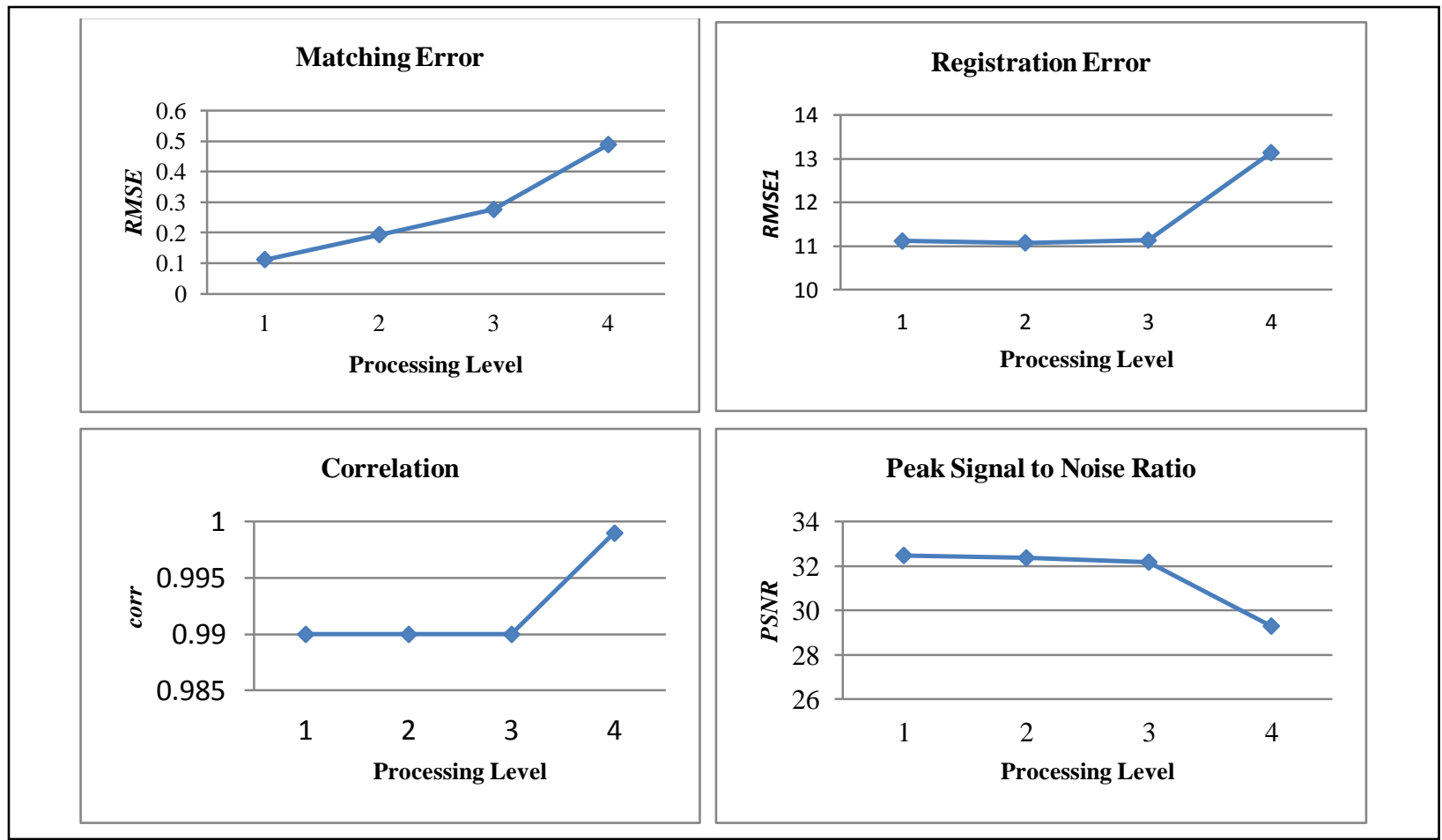

Fig. 12. Registration accuracy (RMSE, RMSE1, corr and PSNR) in different resolution levels, (l=4) for Fig.9.

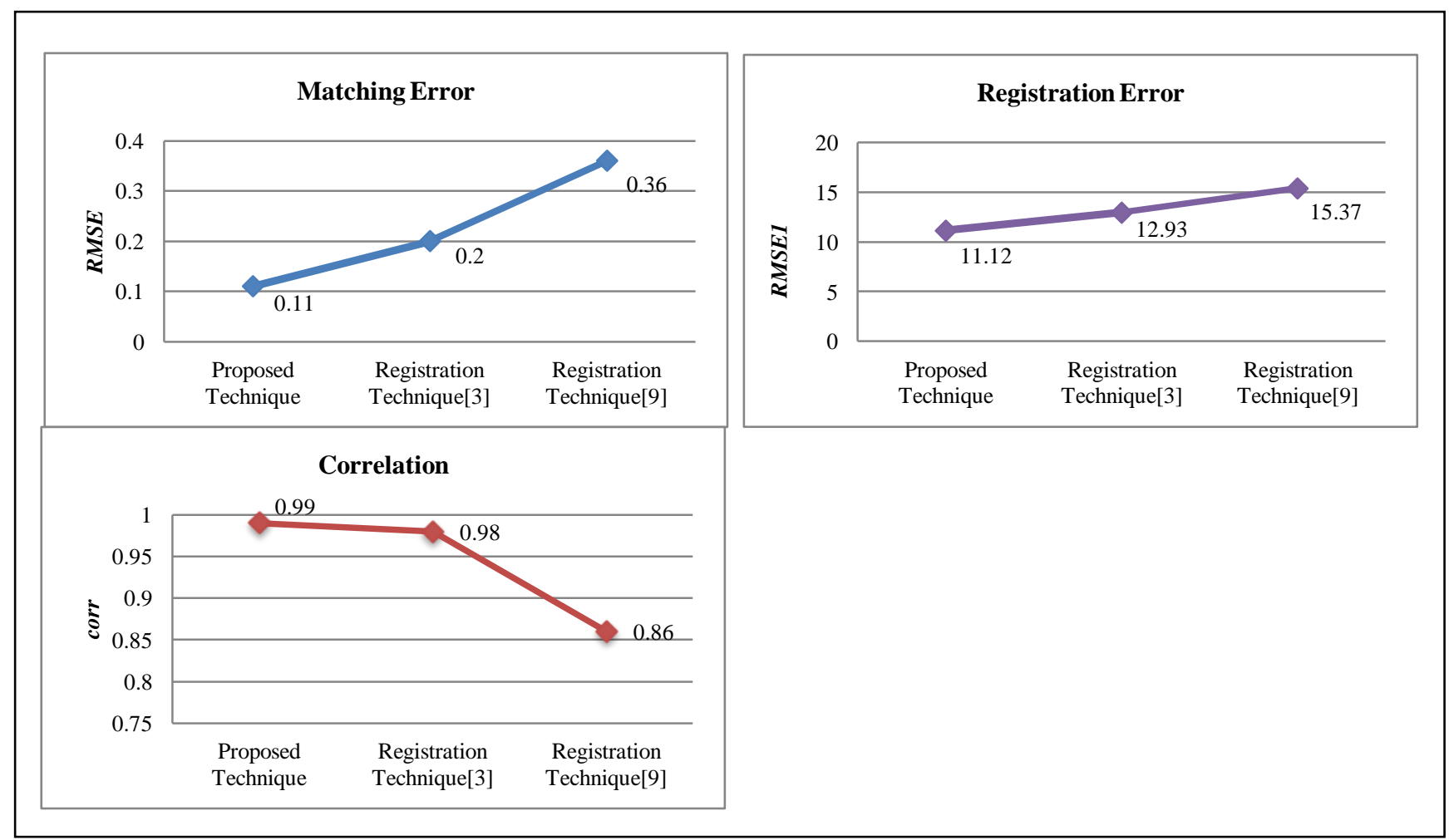

Fig. 13. Comparisons of the registration accuracy ( $R M S E, R M S E 1$ and corr) of the Proposed Technique against the two related Registration Techniques for Fig.9. 


\section{CONCLUSIONS}

In this paper we have presented an automatic registration technique of multi-view, multi-temporal, and multi-spectral remote sensing images based on the Steerable Pyramid Transform and SIFT features that can deal with the large variations of scale, rotation and illumination between images. The median filtering is applied in order to enhance the two input images. The advantage of the proposed technique lies in its ability to increase the number of matched points using the developed SIFT neighborhood matching method and to overcome the outliers introduced in the matching using RANSAC algorithm and hence correctly estimate the transformation matrix. The experimental results show that the proposed technique returns better performance for large scale variations, rotation and intensity changes as compared to the two other related image registration techniques.

\section{References}

[1] D. Suma, S. Vikas, and Sh. Bhudev, "Remote sensing image registration techniques: a survey," In: Proc ISISP, Vol. 6134.P. 103-12, 2010.

[2] Z. Barbra, and F. Jan, "Image registration methods: a survey," Image and Vision Computing 21, 977-1000, 2003.

[3] L. Xiangzeng, T. Zheng, C. Chunyan, and F. Huijing, "Multiscale registrations of remote sensing image using robust SIFT features in steerable-domain," The Egyptian Journal of Remote Sensing and Space Sciences, 2011

[4] M. Nagham, F. Abou-Chadi, and S. Kishk, "Wavelet-based image registration techniques: a study of performance," IJCSNS International Journal of Computer Science a 188 and Network Security, Vol.11 No.2, 2011.

[5] N. Haidawati, S. Vladimir, and M. Stephen, "Image registration for super resolution using scale invariant feature transform, belief propagation, and random sampling consensus," European Signal Processing Conference (EUSIPCO-ISSN 2076-1465, 2010).

[6] L. Sang, "A coarse-to-fine approach for remote-sensing image registration based on a Local method," International Journal on Smart Sensing and Intelligent Systems Vol. 3, No. 4, 2010.

[7] H. Mahmudul, J. Xiuping, R-K. Antonio, Z. Jun, P. Mark, "Multispectral remote sensing image registration via spatial relationship analysis on SIFT keypoints," IEEE 978-1-4244-9566-5.

[8] M. Fatiha, El.M. Miloud, and T. Nasreddine, "A rigid image registration based on the Nonsubsampled Contourlet transform and genetic algorithms," Sensors 2010, 10, 8553-8571, doi: 10.3390/s100908553, 2010.

[9] Z. Yi, C. Zhiguo, and X. Yang," Multi-spectral remote image registration based on SIFT," IEEE Electronics Letter, Vol. 44, No. 2, pp. 107-108, 2008.

[10] Y. Le, Z. Dengrong, and H. Eun-Jung, "A fast and fully automatic registration approach based on point features for multi-source remotesensing images," Computers and Geosciences 34. 838- 848, 2008.

[11] H. Gang and Z. Yun, "Wavelet-based image registration technique for high resolution remote sensing images," Computers \& Geosciences 34.1708-1720, 2008.

[12] F. Leila, C. Max, K. Thales, C. Emiliano, and S. Felipe, "Multitemporal image registration based on multiresolution decomposition," ISSN 1808-0936, 2008.

[13] B. Shirin and Sh. Kasaei, "Contourlet-based edge extraction for image registration," Iranian Journal of Electrical \&Electronic Engineering, Vol. 4, No. 1\&2, 2008.

[14] F. William and A. Edward, "The design and use of steerable filters," IEEE Transactions on Pattern Analysis and Machine Intelligence 13, 891-907, 1991.

[15] L. David, "Distinctive image features form scale-invariant keypoints," International Journal of Computer Vision 60, 91-110, 2004.

[16] F. Martin and B. Robert, "Random sample consensus: a paradigm for model fitting with applications to image analysis and automated cartography," Communications of the ACM, Vol. 24, No. 6, pp. 381395, 1981. 